Bull. Austral. Math. Soc.

VoL. 58 (1998) [261-270]

\title{
JONES POLYNOMIALS OF PERIODIC KNOTS
}

\author{
Yonguu Bae, Young Kon Kim and Chan-Young Park
}

We calculate the Zulli's matrix of a periodic knot and give some necessary conditions for the Jones polynomial of a periodic knot, which are slightly different from Yokota's result.

\section{INTRODUCTION}

A knot $\widetilde{K}$ is said to have period $r>1$, if there exists an orientation preserving homeomorphism $f$ on $S^{3}$ of order $r$ which preserves $\widetilde{K}$ with $\operatorname{Fix}(f)=\left\{x \in S^{3} \mid f(x)=\right.$ $x\} \cong S^{1}$ and $\operatorname{Fix}(f) \cap \widetilde{K}=\emptyset$.

By the positive solution of the Smith conjecture, $\operatorname{Fix}(f)$ is unknotted. Let $\Sigma^{3}=S^{3} / f$ be the quotient space under $f$. Since $\operatorname{Fix}(f)$ is unknotted, $\Sigma^{3}$ is again a 3 -sphere, $\widetilde{K} / f$ is a knot in $\Sigma^{3}$ and $S^{3}$ is an $r$-fold cyclic covering space of $\Sigma^{3}$ branched along Fix $(f)$. Let $\psi: S^{3} \longrightarrow \Sigma^{3}$ be the covering projection map. Denote $\psi(\widetilde{K})=K$ and call it the factor knot of $\widetilde{K}$. Note that $K$ is a knot in the 3 -sphere $\Sigma^{3}$, so we may assume that $K$ is also a knot in $S^{3}$.

Notice that we may have knot diagrams $D(\widetilde{K})$ and $D(K)$ of $\widetilde{K}$ and $K$ respectively, which satisfy the following commutative diagram

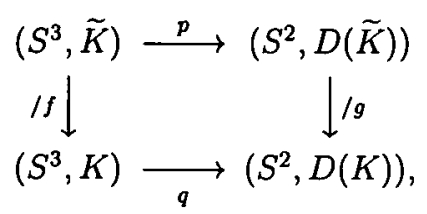

where $g$ is the restriction of $f$ to $S^{2}$ and $p$ and $q$ are regular projections indicating the knot diagrams $D(K)$ and $D(\widetilde{K})$, respectively.

In this paper, we shall not distinguish the notations for a knot and its diagram, so $K$ will represents a knot or its diagram. Notice that the knot diagram $\widetilde{K}$ consists of $r$ periodic sections, each of which gives us the diagram $K$ of the factor knot. Then we

Received 11th February, 1998

This work was partially supported by the Basic Science Research Institute Program, Ministry of Education, 1997, Project No. 97-1409 and TGRC.

Copyright Clearance Centre, Inc. Serial-fee code: 0004-9729/98 \$A2.00+0.00. 
can enumerate the crossings of the knot diagram $\widetilde{K}$ as follows. Let $c_{1}, c_{2}, \cdots, c_{n}$ be the crossings in the first periodic section of $\widetilde{K}$. Then

$$
g^{k}\left(c_{i}\right)=c_{i+r k},(i=1,2, \cdots, n, k=0,1, \cdots, r-1)
$$

represent all the crossings of $\widetilde{K}$. Here we can identify the knot diagram $K$ with the factor of the first periodic section of the knot diagram $\widetilde{K}$ so that $c_{1}, c_{2}, \cdots, c_{n}$ also represent the crossings of $K$.

Now we introduce the definition of the Zulli's matrix of a knot diagram.

Given a knot diagram $K$ with the crossings $c_{1}, c_{2}, \cdots c_{n}$, give any orientation to $K$. The Zulli's matrix of the knot diagram $K$ is the $n \times n$ matrix $T=\left(T_{i j}\right)$ over $\mathbf{Z}_{2}$ defined as follows. For $i \neq j, T_{i j}$ is defined to be the number of times $(\bmod 2)$ that a traveller passes through crossing $c_{i}$ while making the following trip - the traveller begins on the overcrossing $c_{j}$ with the given direction until he returns to the undercrossing $c_{j}$. For $i=j, T_{i i}$ is defined as follows

$$
T_{i i}= \begin{cases}1 & \text { if the crossing sign of } c_{i} \text { is }+1 \\ 0 & \text { otherwise }\end{cases}
$$

A state of the knot diagram $K$ is defined to be a function $S$ from the set of all crossings of $K$ to $\{A, B\}$, that is, a choice, at each crossing $c_{i}$, of a label $A$ or $B$. Let $\mathcal{S}(K)$ denote the set of the states of the knot diagram $K$. Let $S \in \mathcal{S}(K)$ be the state obtained from the state $A A \cdots A$ by exchanging the labels in the positions $c_{i_{1}}, c_{i_{2}}, \cdots, c_{i_{m}}$. Let us denote $T_{S}$ to be the matrix obtained from the matrix $T$ as follows.

$$
\operatorname{ent}_{i j}\left(T_{S}\right)= \begin{cases}1-\operatorname{ent}_{i i}(T), & i=i_{1}, i_{2}, \cdots i_{m}, \\ \operatorname{ent}_{i j}(T), & \text { otherwise. }\end{cases}
$$

Given a matrix $T$, let $n(T)$ denote the nullity of the matrix $T$.

For a knot $K$, the Jones polynomial $V_{K}(t)$ of $K$ is obtained from the Kauffman polynomial

$$
P_{K}(A)=\left(-A^{-3}\right)^{w(K)} \sum_{S} A^{A(S)-B(S)}\left(-A^{2}-A^{-2}\right)^{\sharp(K \mid S)-1}
$$

by putting $A=t^{-1 / 4}$, where $w(K)$ is the writhe of the knot $K, A(S), B(S)$ are the numbers of $A, B$ values in the state $S$, respectively, and $\sharp(K \mid S)$ is the numbers of circles in the split-open diagram $K \mid S$. (See Kaufman [1, 2], Murasugi [3] and Zulli [6].) In [6], Zulli proved that $n\left(T_{S}\right)=\sharp(K \mid S)-1$. We shall use the following notation

$$
A^{A(S)-B(S)}=\operatorname{Coeff}(S) \quad \text { and } \quad d=-\left(A^{2}+A^{-2}\right),
$$

for simplicity. Then the above equation can be simplified to

$$
P_{K}(A)=\left(-A^{-3}\right)^{w(K)} \sum_{S} \operatorname{Coeff}(S) d^{n\left(T_{S}\right)} .
$$




\section{Main Results}

THEOREM 1. Suppose that $\widetilde{K}$ is an $r$-periodic oriented knot diagram which has the factor knot diagram $K$ and $T$ is the Zulli's matrix of $K$. Then the Zulli's matrix of $\widetilde{K}$ is the blockwise circulant matrix $\widetilde{T}$ of the form

$$
\widetilde{T}=\left[\begin{array}{cccc}
T_{1} & T_{r} & \cdots & T_{2} \\
T_{2} & T_{1} & \cdots & T_{3} \\
\cdots & \cdots & \cdots & \cdots \\
T_{r-1} & T_{r-2} & \cdots & T_{r} \\
T_{r} & T_{r-1} & \cdots & T_{1}
\end{array}\right]
$$

where each $T_{k}$ is an $n \times n$ matrix such that $T_{1}$ is symmetric, $T_{k}={ }^{t}\left(T_{r-k+2}\right)$ the transposed matrix of $T_{r-k+2}(2 \leqslant k \leqslant r, r \geqslant 2)$ and

$$
\sum_{k=1}^{r} \operatorname{ent}_{i j}\left(T_{k}\right)=\operatorname{ent}_{i j}(T)
$$

where ent $t_{i j}(A)$ denotes the $(i, j)$-entry of a matrix $A$.

Proof: From the definition of the Zulli's matrix, it is obvious that $T_{k}$ is the $n \times n$ matrix whose $i j$-entry $\operatorname{ent}_{i j}\left(T_{k}\right)(j=1,2, \cdots, n)$ is the number of times $(\bmod 2)$ passing through the crossing $c_{i+r k}$ in the $k$-th periodic section from the overcrossing of $c_{j}$ to the undercrossing of $c_{j}$ along the orientation of the knot diagram $K$. Since $g^{k}\left(c_{j}\right)=c_{j+r k}$, ent $_{i j}(\widetilde{T})=\operatorname{ent}_{i+r k j+r k}(\widetilde{T})$. Thus $\widetilde{T}$ has the form in (1). Since $\widetilde{T}$ is symmetric, $T_{1}$ is symmetric and $T_{k}={ }^{t}\left(T_{r-k+2}\right)$. Since $K$ and $\widetilde{K}$ are knots and $g^{k}\left(c_{j}\right)=c_{j+r k}$, for each $j=1,2, \cdots, r n$,

$$
\sum_{i=1}^{r n} \operatorname{ent}_{i j}(\widetilde{T})=\sum_{i=1}^{n} \operatorname{ent}_{i j}(T), \text { for } i=1,2, \cdots, n
$$

THEOREM 2 For an odd prime $r$, let $\widetilde{K}$ be an $r$-periodic knot with a factor knot $K$ and $f$ be the periodic map on $S^{3}$ realising the r-periodic knot $\widetilde{K}$.

(1) If $\operatorname{lk}(\operatorname{Fix}(f), \tilde{K}) \equiv 1(\bmod 2)$, then

$$
P_{\widetilde{K}}(A) \equiv\left[P_{K}(A)\right]^{r} \quad\left(\bmod r, \lambda_{r}(A)\right) .
$$

(2) If $\operatorname{lk}(\operatorname{Fix}(f), \widetilde{K}) \equiv 0(\bmod 2)$, then

$$
P_{\widetilde{K}}(A) \equiv d^{r-1}\left[P_{K}(A)\right]^{r} \quad\left(\bmod r, \lambda_{r}(A)\right)
$$

Here, $\lambda_{r}(A)$ is the polynomial defined by $\lambda_{r}(A)=A^{8 r}-A^{4(r+1)}-A^{4(r-1)}+1$. 


\section{Lemmas and the Proof of Theorem 2}

LEMMA 1 For an odd prime $r$, let $\widetilde{K}$ be an $r$-periodic knot with a factor knot $K$ and $f$ be the periodic map on $S^{3}$ realising the $r$-periodic knot $\widetilde{K}$. Then

$$
w(\tilde{K})=r w(K) .
$$

Proof: Since $\widetilde{K}$ consists of $r$ periodic sections each of which gives us the diagram $K$ in the quotient, Lemma 1 immediately follows from the definition of $w(K)$.

LemMa 2 For an odd prime $r$, let $\widetilde{K}$ be an $r$-periodic knot with a factor knot $K$ and $f$ be the periodic map on $S^{3}$ realising the r-periodic knot $\widetilde{K}$. Let $\widetilde{S}=S_{1} S_{2} \cdots S_{r} \in \mathcal{S}(\widetilde{K})$, where $S_{i}$ is the state in the $i$-th periodic section of the knot diagram $\widetilde{K}$. If $S_{i} \neq S_{j}$ for some $i, j$, then

$$
\sum_{\tilde{S} \in \mathcal{S}(\tilde{K}), S_{i} \neq S_{j}} \operatorname{Coeff}(\tilde{S}) d^{n\left(\tilde{T}_{\tilde{s}}\right)} \equiv 0 \quad(\bmod r)
$$

Proof: Given the state $\widetilde{S}=S_{1} S_{2} \cdots S_{r} \in \mathcal{S}(\widetilde{K})$ as above, let

$$
\begin{aligned}
& \widetilde{S}_{1}=S_{1} S_{2} \cdots S_{r-1} S_{r}=\widetilde{S} \\
& \widetilde{S}_{2}=S_{2} S_{3} \cdots S_{r} S_{1} \\
& \cdots \quad \cdots \quad \cdots \quad \cdots \\
& \tilde{S}_{r}=S_{r} S_{1} \cdots S_{r-2} S_{r-1} .
\end{aligned}
$$

$S_{i} \neq S_{j}$ as the states of the factor knot $K$ for some $i, j, \widetilde{S}_{1}, \widetilde{S}_{2}, \cdots, \widetilde{S}_{r}$ are all distinct. We have

$$
n\left(\widetilde{T}_{\widetilde{S}_{1}}\right)=n\left(\widetilde{T}_{\widetilde{S}_{2}}\right)=\cdots=n\left(\widetilde{T}_{\widetilde{S}_{r}}\right)
$$

and

$$
\operatorname{Coeff}\left(\widetilde{S}_{1}\right)=\operatorname{Coeff}\left(\widetilde{S}_{2}\right)=\cdots=\operatorname{Coeff}\left(\widetilde{S}_{r}\right)
$$

Hence we have

$$
\sum_{\tilde{S} \in \mathcal{S}(\tilde{K}), S_{i} \neq S_{j}} \operatorname{Coeff}(\tilde{S}) d^{n\left(\tilde{T}_{\tilde{S}}\right)} \equiv 0 \quad(\bmod r)
$$

LEMMA 3 For an odd prime $r$, let $\widetilde{K}$ be an $r$-periodic knot with a factor knot $K$ and $f$ be the periodic map on $S^{3}$ realising the r-periodic knot $\widetilde{K}$. Let $\widetilde{S}=S S \cdots S \in \mathcal{S}(\widetilde{K})$ with $S \in \mathcal{S}(K)$. Then

$$
n\left(\widetilde{T}_{\bar{S}}\right) \equiv n\left(T_{S}\right) \quad(\bmod r-1)
$$


Proof: Let $\widetilde{S}=S S \cdots S \in \mathcal{S}(\widetilde{K})$ be a state of $\widetilde{K}$ and let $D_{1}, D_{2}, \cdots, D_{k}$ be the circles in the diagram $\widetilde{K} \mid \widetilde{S}$. For each $D_{i}, 1 \leqslant i \leqslant k$, define an $r n \times 1$ matrix $\widetilde{R}_{i}=\widetilde{R}_{i}(\widetilde{S})$ by setting

$$
\operatorname{ent}_{j 1}\left(\widetilde{R}_{i}\right)= \begin{cases}1, & \text { if } D_{i} \text { passes through the crossing } c_{j}, \\ 0, & \text { otherwise. }\end{cases}
$$

Since $f(\widetilde{K} \mid \widetilde{S})=\widetilde{K} \mid \widetilde{S}$, for each $i=1,2, \cdots, k$, either the circles $D_{i}$ are all the same ; $D_{i}=g\left(D_{i}\right)=g^{2}\left(D_{i}\right)=\cdots=g^{r-1}\left(D_{i}\right)$ or the circles $D_{i}, g\left(D_{i}\right), g^{2}\left(D_{i}\right), \cdots, g^{r-1}\left(D_{i}\right)$ are all distinct circles in the diagram $\tilde{K} \mid \tilde{S}$.

Let

$$
\widetilde{R}_{i}=\left[\begin{array}{c}
R_{i 1} \\
R_{i 2} \\
\cdots \\
R_{i r}
\end{array}\right]
$$

where $R_{i j}$ is the $n \times 1$ matrix such that $\operatorname{ent}_{l 1}\left(R_{i j}\right)=\operatorname{ent}_{r(j-1)+l, 1}\left(\widetilde{R}_{i}\right)$, for $1 \leqslant l \leqslant n$.

The matrix $\widetilde{R}_{i}$ is said to be Type $I$ if $R_{i 1}=R_{i 2}=\cdots R_{i r}$. Otherwise we say that $\widetilde{R}_{i}$ is of type II. Note that if $\widetilde{R}_{i}$ is of Type II, then

$$
\left[\begin{array}{c}
R_{i 1} \\
R_{i 2} \\
\cdots \\
R_{i r}
\end{array}\right],\left[\begin{array}{c}
R_{i 2} \\
R_{i 3} \\
\cdots \\
R_{i 1}
\end{array}\right], \cdots,\left[\begin{array}{c}
R_{i r} \\
R_{i 1} \\
\cdots \\
R_{i r-1}
\end{array}\right]
$$

are all distinct.

Let

$$
\begin{aligned}
& k_{1}=\text { the number of the matrices of type I, } \\
& k_{2}=\text { the number of the matrices of type II. }
\end{aligned}
$$

Notice that $k=k_{1}+k_{2}$ and that $r$ divides $k_{2}$.

For each $\widetilde{R}_{i}(i=1,2, \cdots, k)$, define an $n \times 1$ matrix $R_{i}=R_{i}(\widetilde{S})$ by setting

$$
R_{i}=\sum_{j=1}^{r} R_{i j}
$$

Then, for a fixed $\widetilde{S} \in \mathcal{S}(\widetilde{K})$, the cardinality $\left|\left\{R_{i} \mid i=1,2, \cdots k\right\}\right|=k_{1}+k_{2} / r$.

Zulli showed in [6] that $\left\{\widetilde{R}_{1}, \widetilde{R}_{2}, \cdots, \widetilde{R}_{k-1}\right\}$ forms a basis for $\operatorname{ker}\left(\widetilde{T}_{\tilde{S}}\right)$, where $\operatorname{ker}\left(\widetilde{T}_{\tilde{S}}\right)$ denotes the kernel of the matrix of $\tilde{T}_{\bar{S}}$ over $Z_{2}$. Now, we claim that the set $\left\{R_{i} \mid i \neq k\right\}$ is a basis for $\operatorname{ker}\left(T_{\widetilde{s}}\right)$. 
By Theorem 1,

$$
\widetilde{T}_{\widetilde{S}}=\left[\begin{array}{cccc}
T_{1} & T_{r} & \cdots & T_{2} \\
T_{2} & T_{1} & \cdots & T_{3} \\
\cdots & \cdots & \cdots & \cdots \\
T_{r-1} & T_{r-2} & \cdots & T_{r} \\
T_{r} & T_{r-1} & \cdots & T_{1}
\end{array}\right]
$$

with $T_{S}=\sum_{i=1}^{r} T_{i}$, and

$$
T_{S} R_{i}=\left(T_{1}+T_{2}+\cdots+T_{r}\right)\left(R_{i 1}+R_{i 2}+\cdots+R_{i r}\right)=0
$$

for

$$
\left[\begin{array}{cccc}
T_{1} & T_{r} & \cdots & T_{2} \\
T_{2} & T_{1} & \cdots & T_{3} \\
\cdots & \cdots & \cdots & \cdots \\
T_{r-1} & T_{r-2} & \cdots & T_{r} \\
T_{r} & T_{r-1} & \cdots & T_{1}
\end{array}\right]\left[\begin{array}{c}
R_{i 1} \\
R_{i 2} \\
\cdots \\
R_{i r-1} \\
R_{i r}
\end{array}\right]=0
$$

Thus, $R_{i} \in \operatorname{ker}\left(T_{S}\right)$

To show that the set $\left\{R_{i} \mid i \neq k\right\}$ generates $\operatorname{ker}\left(T_{S}\right)$, assume that $T_{S} R=0$ for some $n \times n$ matrix $R$. Then

$$
\left[\begin{array}{cccc}
T_{1} & T_{r} & \cdots & T_{2} \\
T_{2} & T_{1} & \cdots & T_{3} \\
\cdots & \cdots & \cdots & \cdots \\
T_{r-1} & T_{r-2} & \cdots & T_{r} \\
T_{r} & T_{r-1} & \cdots & T_{1}
\end{array}\right]\left[\begin{array}{c}
R \\
R \\
\cdots \\
R \\
R
\end{array}\right]=\left[\begin{array}{c}
\sum_{i=1}^{r} T_{i} R \\
\sum_{i=1}^{r} T_{i} R \\
\cdots \\
\sum_{i=1}^{r} T_{i} R \\
\sum_{i=1}^{r} T_{i} R
\end{array}\right]=\left[\begin{array}{c}
0 \\
0 \\
\cdots \\
0 \\
0
\end{array}\right]=0 .
$$

Thus,

$$
\left[\begin{array}{l}
R \\
R \\
\cdots \\
R \\
R
\end{array}\right] \in \operatorname{ker}\left(\widetilde{T}_{\widetilde{s}}\right)
$$


and hence

$$
\begin{aligned}
{\left[\begin{array}{c}
R \\
R \\
\cdots \\
R \\
R
\end{array}\right]=} & c_{1} \widetilde{R}_{1}+c_{2} \widetilde{R}_{2}+\cdots+c_{k-1} \widetilde{R}_{k-1} \\
= & {\left[\begin{array}{c}
c_{1} \widetilde{R}_{11}+c_{2} \widetilde{R}_{21}+\cdots+c_{k-1} \widetilde{R}_{k-1,1} \\
c_{1} \widetilde{R}_{12}+c_{2} \widetilde{R}_{22}+\cdots+c_{k-1} \widetilde{R}_{k-1,2} \\
\cdots \cdots \cdots \cdots \cdots \cdots \cdots \cdots \\
c_{1} \widetilde{R}_{1 r-1}+c_{2} \widetilde{R}_{2 r-1}+\cdots+c_{k-1} \widetilde{R}_{k-1, r-1} \\
c_{1} \widetilde{R}_{1 \tau}+c_{2} \widetilde{R}_{2 r}+\cdots+c_{k-1} \widetilde{R}_{k-1, r}
\end{array}\right], }
\end{aligned}
$$

for some $c_{1}, c_{2}, \cdots, c_{k-1} \in \mathbf{Z}_{2}$. Thus

$$
\begin{aligned}
r R & =c_{1} \sum_{i=1}^{r} R_{1 i}+c_{2} \sum_{i=1}^{r} R_{2 i}+\cdots+c_{k-1} \sum_{i=1}^{r} R_{k-1, i} \\
& =c_{1} R_{1}+c_{2} R_{2}+\cdots+c_{k-1} R_{k-1},
\end{aligned}
$$

so that $R=\sum_{i \neq k} c_{i} R_{i}$, in $\mathbf{Z}_{2}$.

Finally, we want to show that the set $\left\{R_{i} \mid i \neq k\right\}$ is linearly independent. To do this, assume that $c_{1} R_{1}+c_{2} R_{2}+\cdots+c_{k-1} R_{k-1}=0$ with $c_{1}, c_{2}, \cdots, c_{k-1} \in \mathbf{Z}_{2}$. If the matrix $\widetilde{R}_{k}$ is of type I, then clearly,

$$
c_{1} \widetilde{R}_{1}+c_{2} \widetilde{R}_{2}+\cdots+c_{k-1} \widetilde{R}_{k-1}=0,
$$

and all the $r$ matrices obtained from $\widetilde{R}_{i}$ have the same coefficient.

If the matrix $\widetilde{R}_{k}$ is of type II, then $\sum c_{i} \widetilde{R}_{i}=0$, where the sum runs over all matices and the $r$ matrices obtained from $\widetilde{R}_{k}$ have coefficient 0 and the other $r$ matrices obtained from $\widetilde{R}_{i}(i \neq k)$ have the same coefficient $c_{i}$. Thus, in each case, we have $c_{1}=c_{2}=\cdots=$ $c_{k-1}=0$.

We have proved $n\left(T_{S}\right)=k_{1}+k_{2} / r-1$, and hence

$$
\begin{aligned}
n\left(\widetilde{T}_{\tilde{S}}\right) & =k_{1}+k_{2}-1 \\
& =r k_{1}+k_{2}-r-(r-1)\left(k_{1}-1\right) \\
& =r\left(k_{1}+k_{2} / r-1\right)-(r-1)\left(k_{1}-1\right) \\
& =r n\left(T_{S}\right)-(r-1)\left(k_{1}-1\right) \\
& =n\left(T_{S}\right) \quad(\bmod r-1) .
\end{aligned}
$$

LEMMA 4 For an odd prime $r$, let $\widetilde{K}$ be an $r$-periodic knot with a factor knot $K$ and $f$ be the periodic map on $S^{3}$ realising the r-periodic knot $\widetilde{K}$. Let $k_{1}$ be the integer defined in (2). Then

$$
\operatorname{lk}(\operatorname{Fix}(f), \widetilde{K}) \equiv k_{1} \quad(\bmod 2)
$$


Proof: If $D$ is a circle in $\widetilde{K} \mid \widetilde{S}$ such that $g(D) \neq D$, then clearly $D \cap\{(s, \theta) \mid s>$ $0, \theta=(2 \pi / s) t\}$ is even, for $t=1,2, \cdots, r$.

Now we are going to prove Theorem 2.

Proof of TheOREM 2: First, assume that

$$
\operatorname{lk}(\operatorname{Fix}(f), \tilde{K}) \equiv 1 \quad(\bmod 2)
$$

If $\widetilde{S}=S S \cdots S \in \mathcal{S}(\widetilde{K})$ with $S \in \mathcal{S}(K)$, then, by Lemmas 3 and $4, k_{1}$ is odd and

$$
n\left(\widetilde{T}_{\widetilde{S}}\right)=r n\left(T_{S}\right)-2(r-1) k, \text { for some } k \in \mathbf{Z} .
$$

Clearly, $\operatorname{Coeff}(\widetilde{S})=(\operatorname{Coeff}(S))^{r}$ and

$$
\begin{aligned}
d^{n\left(\tilde{T}_{\tilde{s}}\right)} & =d^{r n\left(T_{S}\right)-2(r-1) k} \\
& \equiv\left(d^{n\left(T_{S}\right)}\right)^{r} \quad\left(\bmod r, d^{2(r-1)}-1\right) \\
& \equiv\left(d^{n\left(T_{S}\right)}\right)^{r} \quad\left(\bmod r, \lambda_{r}(A)\right),
\end{aligned}
$$

for

$$
\begin{aligned}
d^{2(r-1)}-1 & =d^{-2}\left(d^{2 r}-d^{2}\right) \\
& =d^{-2}\left[\left(A^{2}+A^{-2}\right)^{2 r}-\left(A^{2}+A^{-2}\right)^{2}\right] \\
& \equiv d^{-2}\left[\left(A^{2 r}+A^{-2 r}\right)^{2}-\left(A^{2}+A^{-2}\right)^{2}\right] \quad(\bmod r) \\
& =d^{-2}\left(A^{4 r}-A^{4}-A^{-4}+A^{-4 r}\right) \\
& =d^{-2} A^{-4 r} \lambda_{r}(A)
\end{aligned}
$$

Thus

$$
\sum_{\tilde{S}=S S \cdots S \in \mathcal{S}(\tilde{K})} \operatorname{Coeff}(\widetilde{S}) d^{n\left(\tilde{K}_{\tilde{S}}\right)} \equiv \sum_{S \in S(K)}\left(\operatorname{Coeff}(S) d^{n\left(T_{S}\right)}\right)^{r} \quad\left(\bmod r, \lambda_{r}(A)\right),
$$

and hence

$$
\begin{aligned}
\langle\tilde{K}\rangle & =\sum_{\tilde{S} \in \mathcal{S}(\tilde{K})} \operatorname{Coeff}(\widetilde{S}) d^{n\left(\tilde{K}_{\tilde{S}}\right)} \\
& =\sum_{\tilde{S} \in \mathcal{S}(\tilde{K}), S_{i} \neq S_{j}} \operatorname{Coeff}(\tilde{S}) d^{n\left(\tilde{T}_{\tilde{s}}\right)}+\sum_{\tilde{S}=S S \cdots S \in \mathcal{S}(\tilde{K})} \operatorname{Coeff}(\widetilde{S}) d^{n\left(\tilde{K}_{\tilde{s}}\right)} \\
& \equiv \sum_{S \in \mathcal{S}(K)}\left(\operatorname{Coeff}(S)^{r} d^{n\left(T_{S}\right)}\right)^{r} \quad\left(\bmod r, \lambda_{r}(A)\right), \text { by Lemma } 2 \\
& \equiv\left(\sum_{S \in \mathcal{S}(K)} \operatorname{Coeff}(S) d^{n\left(T_{S}\right)}\right)^{r} \quad\left(\bmod r, \lambda_{r}(A)\right) \\
& =\langle K\rangle^{r} .
\end{aligned}
$$

By Lemma 1,

$$
\begin{aligned}
P_{\widetilde{K}}(A) & =\left(-A^{-3}\right)^{w(\tilde{K})}\langle\tilde{K}\rangle \\
& \equiv\left(\left(-A^{-3}\right)^{r w(K)}\langle K\rangle\right)^{r} \quad\left(\bmod r, \lambda_{r}(A)\right) \\
& =\left[P_{K}(A)\right]^{r} \quad\left(\bmod r, \lambda_{r}(A)\right) .
\end{aligned}
$$


Next, assume that

$$
\operatorname{lk}(\operatorname{Fix}(f), \widetilde{K}) \equiv 0 \quad(\bmod 2)
$$

If $\widetilde{S}=S S \cdots S \in \mathcal{S}(\widetilde{K})$ with $S \in \mathcal{S}(K)$, then, by Lemmas 3 and $4, k_{1}$ is even, so

$$
\begin{aligned}
n\left(\tilde{T}_{\tilde{S}}\right) & =r n\left(T_{S}\right)-(r-1)(2 k-1), \text { for some } k \in \mathbf{Z} \\
& =r n\left(T_{S}\right)+(r-1)-2(r-1)(k-1) .
\end{aligned}
$$

Thus

$$
\begin{aligned}
d^{n\left(\tilde{T}_{\tilde{s}}\right)} & =d^{r n\left(T_{S}\right)+(r-1)-2(r-1)(k-1)} \\
& =d^{r-1} d^{r n\left(T_{S}\right)-2(r-1)(k-1)} \\
& \equiv d^{r-1}\left(d^{n\left(T_{S}\right)}\right)^{r} \quad\left(\bmod r, d^{2(r-1)}-1\right) \\
& \equiv d^{r-1}\left(d^{n\left(T_{S}\right)}\right)^{r} \quad\left(\bmod r, \lambda_{r}(A)\right)
\end{aligned}
$$

and hence

$$
\begin{aligned}
\langle\tilde{K}\rangle & =\sum_{\tilde{S} \in \mathcal{S}(\tilde{K})} \operatorname{Coeff}(\widetilde{S}) d^{n\left(\tilde{K}_{\tilde{S}}\right)} \\
& =\sum_{\tilde{S} \in \mathcal{S}(\tilde{K}), S_{i} \neq S_{j}} \operatorname{Coeff}(\widetilde{S}) d^{n\left(\tilde{T}_{\tilde{S}}\right)}+\sum_{\tilde{S}=S S \cdots S \in \mathcal{S}(\tilde{K})} \operatorname{Coeff}(\tilde{S}) d^{n\left(\tilde{K}_{\tilde{S}}\right)} \\
& \equiv \sum_{S \in \mathcal{S}(K)}\left(\operatorname{Coeff}(S)^{r} d^{r-1} d^{n\left(T_{S}\right)}\right)^{r} \quad\left(\bmod r, \lambda_{r}(A)\right), \text { by Lemma } 2 \\
& \equiv d^{r-1}\left(\sum_{S \in \mathcal{S}(K)} \operatorname{Coeff}(S) d^{n\left(T_{S}\right)}\right)^{r} \quad\left(\bmod r, \lambda_{r}(A)\right) \\
& =d^{r-1}(\langle K\rangle)^{r}
\end{aligned}
$$

and by Lemma 1,

$$
\begin{aligned}
P_{\widetilde{K}}(A) & =\left(-A^{-3}\right)^{w(\tilde{K})}\langle\tilde{K}\rangle \\
& \equiv\left(-A^{-3}\right)^{r w(K)} d^{r-1}\langle K\rangle^{r} \quad\left(\bmod r, \lambda_{r}(A)\right) \\
& =d^{r-1}\left[P_{K}(A)\right]^{r} \quad\left(\bmod r, \lambda_{r}(A)\right) .
\end{aligned}
$$

CoRollaRY For an odd prime $r$, let $\widetilde{K}$ be an $r$-periodic knot with a factor knot $K$ and $f$ the periodic map on $S^{3}$ realising the r-periodic knot $\widetilde{K}$.

(1) If $\operatorname{lk}(\operatorname{Fix}(f), \widetilde{K}) \equiv 1(\bmod 2)$, then

$$
V_{\widetilde{K}}(t) \equiv\left[V_{K}(t)\right]^{r} \quad\left(\bmod r, \xi_{r}(t)\right)
$$

(2) If $\operatorname{lk}(\operatorname{Fix}(f), \widetilde{K}) \equiv 0(\bmod 2)$, then

$$
V_{\widetilde{K}}(t) \equiv d^{r-1}\left[V_{K}(t)\right]^{r} \quad\left(\bmod r, \xi_{r}(t)\right)
$$

Here, $\xi_{r}(t)=t^{2 r}-t^{r+1}-t^{r-1}+1$. 


\section{REFERENCES}

[1] L.H. Kauffman, 'State models and the Jones polynomial', Topology 26 (1987), 395-497.

[2] L.H. Kauffman, 'New invariants in the theory of knots', Amer. Math. Monthly 95 (1988), 195-242.

[3] K. Murasugi, 'Jones polynomials and classical conjectures in knot theory', Topology 26 (1987), 187-194.

[4] K. Murasugi, 'Jones polynomials of periodic knots', Pacific J. Math. 131 (1988), 319-329.

[5] Y. Yokota, 'The Jones polynomial of periodic knots', Proc. Amer. Math. Soc. 113 (1991), 889-894.

[6] L. Zulli, 'A matrix for computing the Jones polynomial of a knot', Topology 34 (1995), $717-729$.

Department of Mathematics

College of Natural Sciences

Kyungpook National University

Taegu 702-701

Korea 